\title{
De l'enseignement à l'apprentissage de la littérature ou : des savoirs aux compétences
}

\section{Karl Canvat}

\section{(2) OpenEdition}

1 Journals

\section{Édition électronique}

URL : http://journals.openedition.org/trema/1587

DOI : 10.4000/trema.1587

ISSN : 2107-0997

\section{Éditeur}

Faculté d'Éducation de l'université de Montpellier

\section{Édition imprimée}

Date de publication : 1 octobre 2002

Pagination : 77-92

ISSN : 1167-315X

\section{Référence électronique}

Karl Canvat, « De l'enseignement à l'apprentissage de la littérature ou : des savoirs aux compétences », Tréma [En ligne], 19 | 2002, mis en ligne le 01 octobre 2002, consulté le 19 avril 2019. URL : http:// journals.openedition.org/trema/1587 ; DOI : 10.4000/trema.1587

Ce document a été généré automatiquement le 19 avril 2019

Trema 


\title{
De l'enseignement à l'apprentissage de la littérature ou : des savoirs aux compétences
}

\author{
Karl Canvat
}

« Mais pourquoi les élèves ont-ils du mal à trouver du sens à l'école ? Le savoir leur apparaît souvent déconnecté de son usage, coupé même de la pensée, parce que non relié à un usage opérationnel. On apprend, pensent-ils, pour apprendre, pas forcément pour faire ou pour analyser avec ce que l'on sait. [...] La connaissance leur apparaît comme autant de pièces d'un puzzle

qu'on présenterait en vrac sans jamais avoir à

composer une maquette avec. L'école passe en revue des savoirs démontés que les élèves ont peu fréquemment à utiliser pour construire des cohérences. » (M. Develay, 1996, p. 88). « Ou bien [...] il faut se résigner au fait que ce que l'on apprend à l'école ne sert qu'à réussir à l'école, ou bien il faut convenir que ce qui se forme à

l'école c'est le pouvoir de mettre en correspondance une 'situation qui fait problème', correctement identifiée dans toutes ses dimensions, avec des objets culturels qui permettent de l'assumer et de la dépasser. » (Ph. Meirieu, 1991, pp. 127-128). 


\section{De nouveaux défis}

1 Depuis quelques années, les systèmes d'enseignement de la plu-part des pays industrialisés connaissent une profonde mutation et tentent de répondre à un certain nombre de nouveaux défis (M. Romainville, 1996, 1998, 2001).

- Que faire des « savoirs morts » à l'école, produits par l'extraordinaire développement des savoirs (et leur rapide obsolescence), et qui amènent à s'interroger sur les savoirs clés à faire acquérir par les élèves à chaque niveau ?

- Comment réduire les écarts de scolarité, que la massification a plutôt accentués (A. Prost, 1986) ? En d'autres termes, comment démocratiser véritablement l'école?

- Comment favoriser une culture d'« apprenance », orientée vers les pratiques autonomes et les transferts d'apprentissage, à une époque où les systèmes d'enseignement sont invités à participer au maintien de la compétitivité et à fournir au marché de l'emploi des individus adaptables, « performants » et disposant d'un potentiel d'action?

- Comment sortir de la culture scolaire de l'échec, à l'origine de l'exclusion sociale (M. Crahay, 1996) ?

- Comment redonner, pour les élèves, du sens à l'école (M. Develay, 1996) ?

2 Un changement de modèle éducatif se dessine, qui voue de moins en moins l'école à la transmission d'un ensemble de connaissances, de normes, de représentations, et qui lui assigne une mission d'un tout autre ordre : apprendre à s'autoformer.

La vogue actuelle de la notion de « compétence » dans les programmes et les instructions officielles des systèmes d'enseignement de plusieurs pays (Belgique, Québec, Suisse romande...) n'est sans doute pas étrangère au souci d'apporter des réponses à ces problèmes. Elle traduit aussi l'essoufflement d'un certain nombre de courants pédagogiques - notamment le modèle psychosociologique d'inspiration rogérienne, dominant dans les années 1970, qui privilégiait la relation maître - élève et ne s'interrogeait pas sur la nature des savoirs à transmettre (selon l'aphorisme « apprendre vaut mieux qu'enseigner»), ou la pédagogie formelle des "habiletés intellectuelles » (que résume le slogan « apprendre à apprendre ») dans les années $1980^{1}$ - et la montée du paradigme socioconstructiviste (voir Ph. Jonnaert, C. Vander Borght, 1999) dans le champ des sciences de l'éducation. Elle tente aussi - et peut-être surtout - de dépasser le « dilemme pédagogique », dont on pourrait formuler les termes de la manière suivante :

- tout enseignement suppose une transmission de savoirs ;

- mais il n'y a apprentissage que s'il y a appropriation par le sujet de connaissances qu'il a luimême élaborées (et l'apprentissage étant un acte libre d'un sujet, l'interpellation de cette liberté devient, dès lors, fondamentale²), mobilisables en cas de besoin (" transférables »).

Cette tension n'est pas nouvelle. Elle avait amené J.-J. Rousseau (voir L'Émile) à considérer que la formation intellectuelle se fonde sur la "ruse», qui consiste à rendre l'autre susceptible de désirer ce que nous-mêmes avons décidé qu'il était bon qu'il désirât, parce que cela correspond à ce dont $\mathrm{il} \mathrm{a} \mathrm{besoin} \mathrm{pour} \mathrm{son} \mathrm{développement...}{ }^{3}$

\section{Savoirs et compétences littéraires}

5 Il s'agit d'abord de s'entendre sur ce que l'on entend par «savoirs » et par « compétences ». Les savoirs désignent les savoirs « savants » des disciplines de référence, 
transposés à des fins d'« enseignabilité » et d'«apprenabilité ». C'est donc autour d'eux que prend forme et se développe un projet d'enseignement: en effet, comme le dit en substance, Y. Chevallard, ce qui justifie l'institution scolaire, c'est l'acquisition d'un savoir distinct de celui que l'on peut acquérir dans la vie quotidienne (1985, p. 26 et sqq.).

Les savoirs littéraires scolaires, comme cela a été développé ailleurs (voir notamment $\mathrm{K}$. Canvat et G. Legros, 1997 ; K. Canvat, 2000 a et b), renvoient aux dimensions socioinstitutionnelles, formelles et historiques de la littérature :

- savoirs socio-institutionnels : champ et institutions littéraires, réseau de diffusion rerstreinte/élargie, instances et agents du champ, etc. ;

- savoirs formels : types (narratif, descriptif...), genres (narration fictionnelle, poésie, théâtre...), fiction (intrigue, personnage...), narration (niveaux de narration, voix narrative...), etc. ;

- savoirs historiques : courants, écoles, auteurs...

7 Mais privilégier l'entrée par les savoirs en dehors de toute référence aux problèmes qu'ils permettent de traiter, c'est-à-dire détachés des situations qui leur donnent du sens, revient à définir une logique d'apprentissage qui convient peut-être à de purs «sujets épistémiques ", ouverts et disponibles à l'universel kantien, mais qui ne correspond pas du tout à ce que l'on sait aujourd'hui sur la diversité des apprenants et sur la construction des savoirs en termes d'interactions, de conflits sociocognitifs, de contrat didactique, de méthodes actives et comparatives, de motivation, etc. C'est évacuer complètement les problèmes liés à l'insécurité culturelle, les dimensions sociales et affectives du « rapport au savoir » (voir les travaux de B. Chariot, 1997 et d'E. Bautier et J.-Y. Rochex, 1998) ; c'est, en somme, assez exactement, reconduire l'« épistémocentrisme », dans lequel P. Bourdieu voit le type même de l'« erreur scolastique » (1997), « indifférente aux différences » et préjudiciable aux élèves qui ne sont pas des « héritiers ».

8 Aussi longtemps qu'ils ne sont pas intégrés, les savoirs risquent donc de rester « inertes », c'est-à-dire inutiles. Le problème est ainsi d'en faire des ressources mobilisables, disponibles dans diverses situations, ce qui nécessite que l'on travaille à leur mise en œuvre dans de vrais problèmes et ce, non pas "plus tard", mais à l'école. Cette préoccupation est essentielle pour la discipline littérature, souvent perçue comme " artificielle», " gratuite», « inutile ». Comme le souligne en effet Ph. Perrenoud, «[...] la préoccupation du transfert et de la mobilisation accroitrait [...] le sens du travail et des savoirs scolaires: une partie des élèves apprennent difficilement parce que les savoirs qu'on leur enseigne sont décontextualisés, coupés de leur histoire aussi bien que de toute référence aux pratiques sociales qui s'en servent; ce sont des savoirs morts. À l'école, on devrait d'abord apprendre à penser, à se servir des savoirs pour mener à bien une activité qui met en ceuvre un projet ou répond à un problème et qui, du coup, confronte à des difficultés et à des décisions. » (2000, p. 180). 


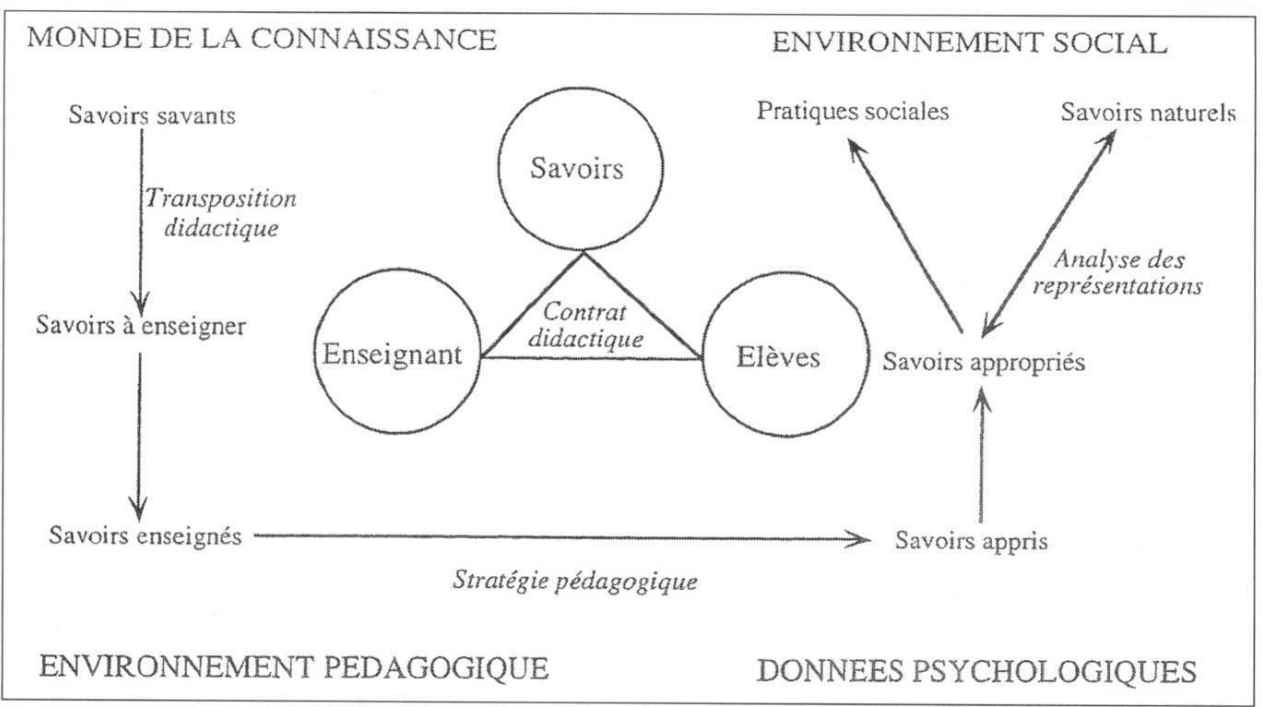

Dans les pratiques dominantes d'enseignement, les savoirs savants sont le point de départ et les savoirs appris, le point d'arrivée. Entre les deux intervient le processus désormais bien connu de la «transposition didactique ».

Les théories de l'apprentissage d'aujourd'hui insistent sur la nécessité d'un cheminement inverse. Le point de départ, ce sont les situations sociales de mobilisation des acquis scolaires (les "pratiques sociales de référence ${ }^{4}$, et le principe d'organisation des apprentissages est la finalisation (on privilégie l'ancrage dans les activités qui ont du sens pour l'élève).

11 C'est ici qu'intervient la notion de "compétence ", notion nomade (elle est issue du monde du travail et de l'entreprise ${ }^{5}$ ) et «techniciste », qui fait l'objet de débats très vifs entre les utilitaristes, qu'ils soient de gauche ( l'école doit donner des moyens d'agir dans et sur le monde ») ou de droite (« l'école doit s'adapter à la demande du marché et à la modernisation économique »), et les défenseurs d'une culture générale, gratuite ${ }^{6}$ (voir Ph. Perrenoud, 1997 ; Raisons éducatives, 1999. Pour une synthèse récente, voir Ch. Bosman, F.-M. Gérard et X. Roegiers, 2000).

Mais qu'est-ce qu'une compétence? La plupart des définitions données par les spécialistes (voir notamment L. Allal, 1999) se rejoignent sur trois paramètres définitionnels. Une compétence :

- comprend plusieurs connaissances mises en relation;

- s'applique à une famille de situations ;

- est orientée vers une finalité (la résolution d'une situation - ou d'une tâche - problème).

Les compétences ont une composante cognitive (ce sont les connaissances déclaratives les savoirs - les connaissances procédurales - les savoir-faire - et les connaissances conditionnelles ou contextuelles), une composante métacognitive (les métaconnaissances et les régulations métacognitives qui interviennent dans la mobilisation et la gestion 
active de la compétence en situation) et une composante socio-affective.

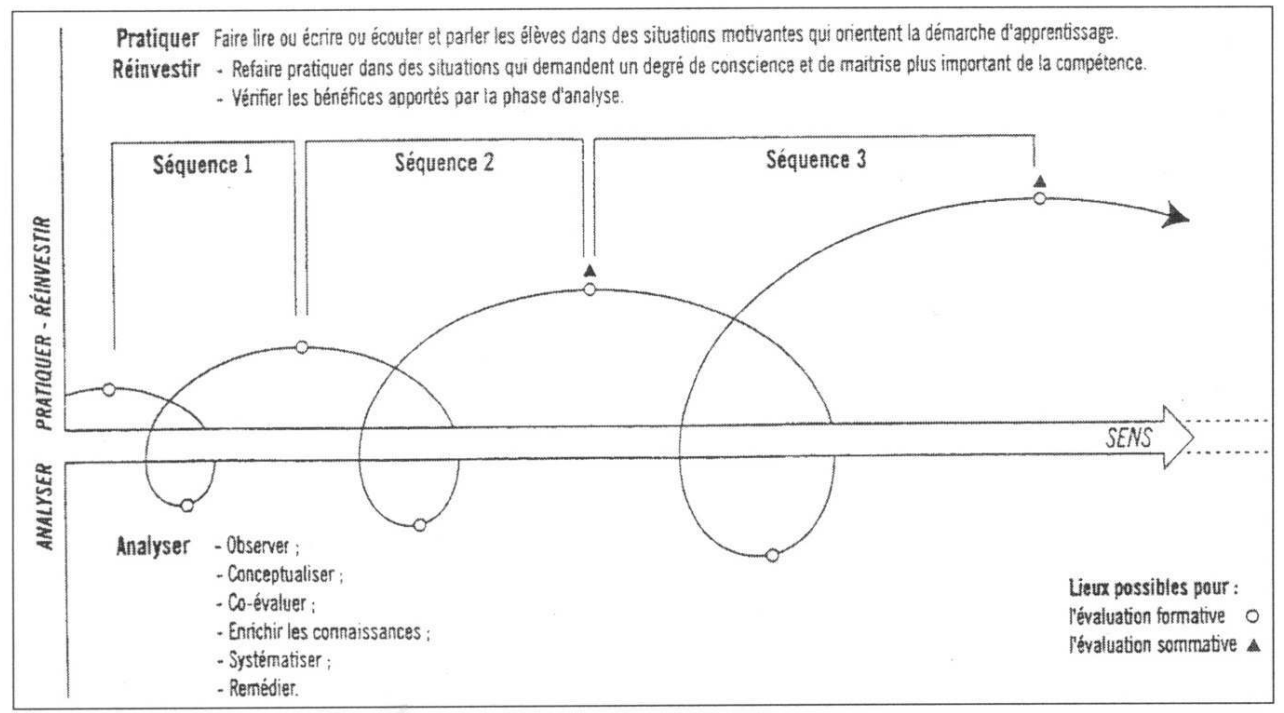

Il importe de préciser, à la lumière des travaux sur la cognition située, qu'une compétence se construit toujours en situation( « la manière dont le sujet apprend fait partie de ce qu'il apprend », disait déjà J. Dewey). On voit que l'on se situe ici résolument dans une perspective constructiviste, puisqu'il s'agit, par la définition de compétences, de déterminer des dispositifs qui permettent une acquisition structurée et progressive de savoirs et de savoir-faire, et, à travers ceux-ci, de rendre l'apprenant plus autonome dans l'accès au savoir et dans la maîtrise des savoir-faire.

Il faut insister sur l'intérêt que présente la définition de compétences dans l'enseignement de la littérature. Pédagogiquement, elle permet un aménagement des tâches et un guidage cohérent de l'apprentissage. En effet, à partir des compétences, on peut construire une programmation de séquences didactiques dans une dialectique de contextualisation - décontextualisation-recontextualisation (pratiquer, analyser, réinvestir).

En outre, le caractère évaluable de la compétence permet à l'enseignant d'intervenir dans le processus d'apprentissage (évaluation diagnostique préalable, constitution de groupes de besoin, régulation en cours d'appropriation, évaluation formative et sommative, remédiations...).

\section{Quelles compétences littéraires ?}

16 À partir de là, on peut évidemment s'interroger sur les pratiques sociales qui pourraient servir de référence à un enseignement-apprentissage de la littérature. La littérature, en effet, ne "sert » à rien. Ou elle sert à tout, elle imprègne (comme l'histoire) le discours social et ses pratiques de référence sont, dès lors, proprement infinies. À nouveau donc : quelles pourraient être les compétences littéraires? Et qu'est-ce qui pourrait faire sens pour l'élève ?7

On pourrait avancer que les compétences littéraires devraient se rapprocher des usages plus réglés, plus institués, plus spécifiés de ceux qui, d'une manière générale, par loisir, par profession ou par vocation, entretiennent un rapport « intéressé » avec la littérature en dehors de l'espace scolaire (les pratiques « ordinaires » et les pratiques « expertes »). 
Cette orientation nécessite, on le devine, la recherche d'une plus grande validité écologique de l'enseignement-apprentissage de la littérature, tout en évitant sa réduction (platement) utilitaire.

18 Il s'agit donc de penser un dispositif qui permette l'articulation des savoirs littéraires avec des compétences communicationnelles, qui permette le tressage d'une double logique d'apprentissage, à la fois culturelle (les savoirs ont une valeur en eux-mêmes) et fonctionnelle (les compétences donnent du sens aux savoirs) ${ }^{8}$.

Trois domaines de compétences communicationnelles apparaissent fondamentaux pour l'apprentissage de la littérature : la lecture, l'écriture et l'oral.

\subsection{L'appropriation lecturale}

\section{La lecture littéraire : retour sur une fausse évidence}

On parle beaucoup aujourd'hui de "lecture littéraire » et la maîtrise de cette habileté apparait souvent comme l'aboutissement de l'enseignement - apprentissage de la littérature (voir J.-L. Dufays, L. Gemenne, D. Ledur, 1996; A. Rouxel, 1997...). Certaines réflexions de E Bourdieu (1987, p. 132-143) conduisent toutefois à s'interroger sur la naturalisation et l'universalisation de ce mode de lecture, en fait très particulier (il s'agit d'une lecture désincarnée et dématérialisée, " pure »), et sur les effets de sa valorisation scolaire - mais aussi sociale. S'appuyant sur les travaux de B. Lahire (1993, 1994), Y. Reuter a d'ailleurs été jusqu'à remettre en question la notion même de "lecture littéraire »: [....] la 'lecture littéraire' est une abstraction, un pré-concept. Mais cela ne l'empêche pas - bien au contraire - de remplir des fonctions précises. Soit, à titre d'exemples : universaliser et intemporaliser la littérature et la lecture littéraire; poser la lecture de la littérature comme une évidence partagée et non interrogeable (ce qui fait apparaître toutes les autres lectures, en tant que lectures ou en tant que lectures sur des objets particuliers comme limitées, spécifiques, réductrices et peu valorisées. » $(1996$, p. 34).

B. Daunay, pour sa part, a montré que l'opposition établie - et depuis, largement diffusée par M. Picard entre «lu » (le lecteur piégé ou complice) et « lectant » (le lecteur critique) (1986; voir aussi V. Jouve, 1992) reproduit un couple d'oppositions simplificateur entre « lecture populaire » et « lecture savante » et que la valorisation subreptice dont celle-ci est l'objet reconduit le point de vue lettré sur la lecture. Il y a là, selon lui, le fondement d'une « culture du mépris » (1999, p. 56), puisque la lecture des « experts» devient l'aune à laquelle se mesure la valeur de toute lecture.

\section{La « posture » (scolaire) de lecture littéraire}

La lecture - toute lecture, mais peut-être davantage celle de la littérature, en tant qu'elle est fiction (J.-M. Schaeffer, 1999) - a inévitablement une fonction «éthico-pratique » (B. Lahire) et psycho-affective, essentielle dans la construction du sujet, particulièrement de l'adolescent (E. Schön et al ; M. Burgos, 1994). Vouloir, par conséquent, évacuer la lecture ordinaire, "naïve ", au profit de la lecture littéraire, "critique ", considérée comme la seule légitime, revient à exclure purement et simplement ce qui est, en fait, le « régime de lecture » normal de la plupart des gens.

Ce problème s'avère d'autant plus sérieux que, selon certaines enquêtes sociologiques récentes, il est l'une des causes de la baisse sensible des pratiques de lecture constatée 
entre le collège et le lycée. En effet, alors que les contenus humains (les problèmes moraux et métaphysiques) constituent, pour la majeure partie des élèves, l'intérêt irremplaçable de la littérature, ce sont les méthodes d'analyse (voir la célèbre « lecture méthodique ») qui sont devenues l'objectif premier de son enseignement. Comme le notent G. Mauger et Cl. F. Poliak, «[...] l'intérêt lettré pour le texte s'est progressivement déplacé du monde représenté vers le dispositif de représentation : l'analyse formelle du texte conçu comme machine linguistique et sémiotique a été peu à peu constituée en idéal-type de la lecture lettrée." (1998, p. 23).

Ce saut qualitatif non seulement serait à l'origine du clivage qui apparaît entre les pratiques de lecture au collège (où domine encore la « foi du charbonnier ») et celles du lycée (où s'installent des "pratiques sans croyance»), mais surtout il accroîtrait considérablement la distance entre les élèves eux-mêmes, comme l'ont noté Ch. Baudelot et $\mathrm{M}$. Cartier : [...] face à la redéfinition de la lecture imposée au lycée, les différents élèves ne vont pas réagir de la même façon. Les mieux adaptés à l'univers scolaire vont s'y convertir, tandis que d'autres, et ils sont de plus en plus nombreux à mesure que se modifie la composition sociale des lycées, s'y déclareront réfractaires. Les premiers sauront aisément jouer du double registre, les autres non. [...] En prenant des distances aussi grandes avec cette forme universelle d'appropriation des livres que constitue la lecture ordinaire, l'enseignement de la littérature au lycée contribue paradoxalement à éloigner du livre tout court des fractions croissantes de la jeunesse d'aujourd'hui, qui n'ont plus rien à voir avec les héritiers d'hier.» (1998, p. 41-43).

Il est donc indispensable de tenir compte des" appropriations non académiques des textes, souvent perçues comme incultes ou primaires ou vulgaires, mais manifestement riches de convictions et de promesses parce que intensément vécues, fût-ce dans l'illusion référentielle 》 (J.M. Privat, 1996, p. 22) et la priorité est d'installer chez les élèves - et ce, le plus tôt possible - la conscience de l'existence de plusieurs postures de lecture (voir D. Bucheton, 1999 ; K. Canvat, 1999. Sur la notion de "posture ", voir la mise au point de M. Rebière, 2001). C'est ce que souligne très fortement M. Burgos:" Non qu'il ne soit indispensable de développer et d'approfondir toujours plus la compréhension du texte, dans ses dimensions linguistiques, narratives, actancielles, etc., mais cette compréhension ne saurait signifier quelque chose pour l'élève qu'à la condition, me semble-t-il, d'être rattachée à une pratique de lecture 'ordinaire', une pratique d'interprétation spontanée, 'naïve' au sens donné par Eco, à la mesure des compétences du lecteur, fruit de ses expériences antérieures, à un moment donné de son discours de vie.»(1992, p. 59).

Mais il est tout aussi indispensable de construire conjointement des comportements de lecteur, d'« instituer " des lecteurs en mettant en place des médiations, culturelles et textuelles (voir J.-M. Privat, 1993, 1995 ; J.-M. Privat, Y. Reuter, 1991 ; J.-M. Privat, M.-Ch. Vinson, 1986, 2000)9.

\subsection{L'appropriation scripturale}

Les pratiques d'écriture devraient être plus étroitement liées à l'enseignement de la littérature qu'elles ne le sont d'ordinaire. On connait l'intérêt des pratiques d'écriture imitation - pastiches, parodies... (voir Pratiques, 1984). D'apparition plus récente (voir les nouveaux programmes de français de 2000-2001), l'« écriture d'invention» permet notamment aux apprenants de s'approprier les connaissances, de les restituer et de développer leur capacité d'expression et leur faculté créative (A. Petitjean, 2001, 2002). 
prenant devrait également avoir régulièrement l'occasion d'exposer sa relation esthétique à une œuvre (imposée ou de son choix), mais aussi de dépasser ce jugement (personnel) de goût et d'argumenter (en raison) un jugement de valeur littéraire. Celui-ci ne peut plus être fondé aujourd'hui sur des bases échappant à toute discussion, sur un sensus communis, encore moins sur une "croyance» (la Beauté...). La connaissance des codes d'écriture, de l'histoire et des lois de fonctionnement du champ littéraire fournit une combinatoire de critères de jugement, nécessaire pour mesurer la part d'originalité et/ou de stéréotypie d'une œuvre ou d'un texte, permettant ainsi d'éviter tant le légitimisme dogmatique ( hors des classiques, point de salut!»), que le relativisme absolu (" une paire de bottes vaut Shakespeare»), et d'accéder à un "point de vue éclairé ». Revenant sur certaines des thèses défendues précédemment ${ }^{10}, \mathrm{G}$. Genette écrit ainsi : [...] la différence entre le point de vue naif et le point de vue éclairé (je ne donne désormais à cet adjectif aucun autre sens que celui de 'non naï', et plus précisément de 'qui-pense-avoirdépassé-le-stade-de-la-naïveté) est incontestablement une différence de degré, ou, si l'on préfere, une différence cumulative et vectorisée: dans le mouvement qui consiste, le cas échéant, à reconnaitre-et-dépasser, l'éclairé est, par définition, situé plus loin, et en un sens plus haut, que le naif, et, tant que l'on se situe dans cette perspective linéaire, il jouit de l'indéniable supériorité psychologique (laissons ici de côté l'aspect sociologique de cette relation, qui n'en manque certes pas) de celui qui connait, ou comprend, l'autre mieux que l'autre ne le connait ou ne le comprend." (1999, p. 226. Car. gr. de l'auteur).

Appropriations lecturale et scripturale devraient donc avoir pour objectif ultime la construction d'une "rationalité esthétique", au sens où l'entend R. Rochlitz: "Sans chercher à réduire l'art dans son ensemble à une forme quelconque de raison, la formule de la 'rationalité esthétique' vise plutôt à cerner la caractéristique des cuvres d'art d'exiger une reconnaissance de leur ambition et de leur degré de réussite, d'être susceptible de critique et de justification argumentée. Si la formule est polémique, elle se dirige plutôt contre une conception qui croit pouvoir discerner la spécificité de l'art dans une relation compensatrice [...] dans laquelle il n'y aurait rien à argumenter. Elle tente, d'autre part, de définir une forme de rationalité qui ne soit applicable qu'à la validité esthétique, analogue à la fois au vrai ou faux dans le domaine de la connaissance et à la justesse normative dans le domaine éthique, mais irréductible à ces types de validité.» (1994, p. 203).

\subsection{L'appropriation orale}

Les activités orales autour de la littérature se limitent beaucoup trop souvent à la présentation d'exposés (sur un auteur, un genre, une période, etc.). L'écoute d'un texte (" audio-livres », C.D., etc.), sa lecture, silencieuse ou à voix haute, constituent autant de moments privilégiés d'appropriation de la littérature, tout comme l'organisation d'un débat (J. Dolz, B. Schneuwly, 1999) ou celle d'un café littéraire (C. Bisenius-Penin, 2001).

Il y aurait lieu également de susciter les prises de paroles des apprenants sur et autour des textes travaillés en classe (E. Non-non, 1995; A. Rouxel, 2001), qui permettent de construire des postures métacognitives, d'acquérir des connaissances, des métalangages, etc. 


\section{Un dispositif de formation par situations et tâches problèmes}

31 Si l'on accepte le postulat, aujourd'hui communément admis, que « l'élève est le centre organisateur d'un savoir qui lui est radicalement hétérogène » (J.-P. Astolfi, 1992, p. 114), il s'agit de s'interroger à présent sur les dispositifs didactiques à mettre en place.

On sait que les différents modèles d'apprentissage qui se sont succédé ne présentent pas tous la même légitimité scientifique ni surtout la même rentabilité didactique. Inspiré des travaux de J. Piaget et de L.S. Vygotsky, le paradigme socioconstructiviste rencontre aujourd'hui une large audience. Grossièrement résumé, il postule que l'individu apprend pour répondre à une question ou pour résoudre un problème qu'il se pose et que les interactions entre les élèves ainsi qu'entre ceux-ci et l'enseignant sont constitutives du processus d'apprentissage (F. Raynal et A. Rieunier, 1997 ; Ph. Jonnaert, C. Vander Borght, 1999).

Selon Ph. Meirieu (1988), les situations d'apprentissage, pour être efficaces, doivent impliquer les apprenants dans la réalisation d'une tâche caractérisée par un obstacle que l'élève ne peut surmonter sans acquérir des connaissances nouvelles : c'est le principe de la situation-problème.

"D'un côté, nous avons ce que, par simplification, nous pouvons appeler la 'pédagogie de la réponse' : elle se contente de livrer des explications correctement agencées, elle fait des leçons qui, sans doute, à terme, permettront de résoudre des problèmes... mais des problèmes que Von rencontrera seulement plus tard. [...]

" À l'autre bout de l'échiquier pédagogique, on trouve ce que, pour simplifier à nouveau, nous nommons les pédagogies du problème : méthodes actives, pédagogies du concret ou du projet, elles se proposent de mettre l'élève face à une tâche susceptible de le mobiliser, et à l'occasion de laquelle on cherchera à lui faire effectuer des apprentissages précis. [...] » Tout l'effort de la pédagogie des situations-problèmes est d'organiser précisément l'interaction pour que, dans la résolution du problème, l'apprentissage s'effectue. Cela suppose que l'on s'assure, à la fois, de l'existence d'un problème à résoudre et de l'impossibilité de résoudre le problème sans apprendre. » (1988, p. 123)

Les avantages de cette conception de l'apprentissage ne sont pas négligeables: les situations-problèmes favorisent l'implication et la motivation des élèves (Ph. Carré, 1999; J.-F Dortier, 1999), elles contribuent au progrès cognitif et au transfert (ou à la mobilisation) des apprentissages (J. Tardif, 1999) et elles impliquent des activités en relation avec les pratiques sociales de référence.

Ce mode d'apprentissage requiert toutefois que soient clairement définies, à l'intention de l'élève, des tâches à résoudre. Comme celle de "situation - problème ", la notion de "tâche - problème » apparaît chez Ph. Meirieu :"Si l'on observe [...] ce qui structure une 'discipline d'enseignement' ", écrit celui-ci, " on est bien obligé de convenir que ce sont d'abord des 'tâches', c'est-à-dire des exercices qui obéissent très largement à des conventions et qui constituent le référent de l'enseignement dispensé ainsi que le moyen d'en évaluer les résultats. » (1991, p. 124-125). 
CHEVAILLIER J.R. et AUDIAT P. : Les textes français. XVIIIe siècle, XIXe siècle - classes de troisième, seconde et première. Paris, Hachette (Les Humanités Françaises), 1929, p. 911.

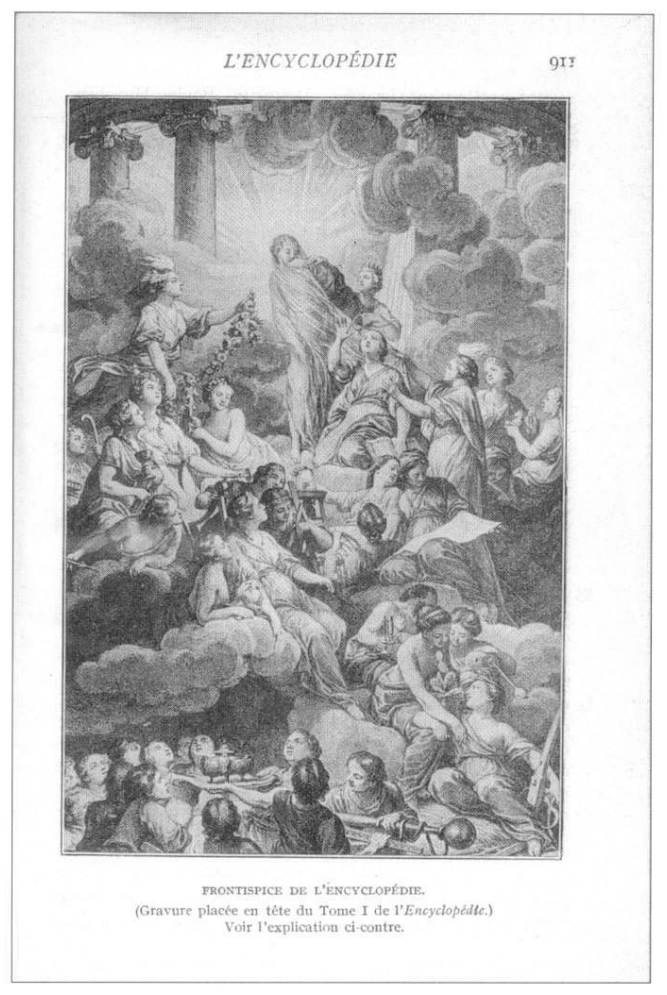

Pour qu'une activité soit une tâche-problème, elle doit permettre à l'enseignant comme à l'apprenant d'accomplir un progrès (notamment cognitif, mais aussi éventuellement affectif, conséquence de l'investissement dans la tâche), elle doit favoriser la contextualisation- décontextualisation-recontextualisation des connaissances (M. Fabre, 1999 ; L. Poirier Proulx, 1999). Mais l'apprentissage ne sera effectif que si le plan de formation littéraire est organisé de manière « concentrico-spi-ralaire », sans rupture, de la maternelle (voir notamment C. Tauve-ron, 2002) à la fin du lycée (voir notamment J.-L. Dumortier, 2001).

\section{Quelques réflexions pour conclure}

Il ne faut pas s'illusionner: la conception d'un dispositif de formation littéraire par compétences ne permettra pas de résoudre tous les problèmes qui se posent à l'enseignement-apprentissage de la littérature. D'abord parce que la littérature n'est pas tout à fait une discipline scolaire comme les autres et qu'elle ne ressortit pas exclusivement à une logique utilitaire, fonctionnaliste. Significativement, G. Le Boterf, pourtant considéré comme la référence dans l'approche par les compétences, s'interrogeait ainsi récemment: Le rôle de l'enseignement général n'est-il pas de développer chez les élèves une variété et une richesse de 'ressources' plutôt que de se focaliser sur l'acquisition de compétences? Une étude approfondie des cuvres littéraires n'est-elle pas, par exemple, un excellent moyen de se préparer à la complexité des situations humaines? La grammaire ne prépare-t-elle pas indirectement à la réflexivité, à la capacité de distanciation? [...] On peut certes définir des compétences en mathématique ou en géographie. Mais ne faut-il pas préserver des 
contributions 'indirectes' en ce qui concerne la littérature, la philosophie, les humanités?» (2000, p. 19)

Dans le même sens, Ph. Perrenoud affirme :» [...] il serait absurde, au nom de l'approche par compétences, de prétendre éliminer des programmes tout savoir qui ne serait pas clairement présenté comme une ressource mobilisable dans l'exercice d'une compétence identifiée et valorisée. » (2000, p. 31).

Ensuite, parce que, derrière tout modèle d'enseignement par compétences, se profile toujours,volens nolens, le modèle behavioriste, dont on connait bien aujourd'hui les limites. En d'autres termes, le risque existe que la focalisation sur les compétences ne conduise à justifier une culture pédagogique de la performance et du conditionnement, que la multiplication et la parcellisation des tâches n'occulte toute saisie des vraies finalités et des véritables enjeux de la formation littéraire. Comme le dit très bien J.-L. Dufays: :...] être compétent en littérature térature ne se limite pas à pouvoir mobiliser des savoirs et des capacités d'écriture, de lecture, d'écoute ou de parole: cela consiste aussi à développer un certain état d'esprit, une attention à certaines caractéristiques des ceuvres, un intérêt pour les livres, pour la poésie, la fiction et le théâtre, c'est-à-dire des attitudes 'gratuites' qui ne sont pas évaluables dans le cadre d'un travail scolaire, mais qui contribuent fortement à éveiller ou à entretenir la motivation à l'égard de la littérature.» (2001, p. 249).

Les visées de la formation littéraire ne sont donc pas plus réductibles à la seule transmission - assimilation de savoirs qu'à la seule construction de compétences. J.-L. Dumortier en a très bien défini les composantes: éducation du goût, exercice du jugement de valeur, développement du sens de l'histoire des faits littéraires, acculturation, émancipation (2000).

41 Les enjeux ne sont pas négligeables : permettre aux apprenants - à tous les apprenants, « experts » et « convers » (M. Burgos, 1992, 1993) - de dépasser l'opinion, de se constituer dans et par le dialogisme, à la recherche d'un socialement partageable; leur permettre, en somme, d'accéder à cette " éthique communicationnelle » (J. Habermas), fondement aujourd'hui plus que jamais indispensable d'une école de la citoyenneté.

42 " Facilitateur ", " entraîneur ", " accompagnateur »... On le devine, c'est un "scénario pour un métier nouveau» qui se dessine pour les enseignants de littérature. Ils y gagneront en partage de responsabilités et sans doute en efficience. À condition d'accepter un changement dans la représentation qu'ils se font de leur métier.

Ce ne sera pas le plus facile...

\section{BIBLIOGRAPHIE}

ALLAL L. : " Acquisition et évaluation des compétences en situation scolaire », in Raison

éducatives, $\mathbf{N}^{\circ}$ 1-2 (L'énigme de la compétence en évaluation), 1999.

ASTOLFI J.-P. : L'école pour apprendre.Paris, ESF éditeur (Pédagogies), 1992. 
BAUDELOT C, CARTIER M., DETREZ C. : Et pourtant, ils lisent... . Paris, Le Seuil (L'épreuve des faits), 1999.

BAUTIER E. : « Rapport aux savoirs et aux lycées des nouveaux lycéens », in Le français aujourd'hui, $\mathrm{N}^{\circ} 115$ (Des changements au lycée),1996.

BAUTIER E.,ROCHEX J.-Y. : L'expérience scolaire des nouveaux lycéens. Démocratisation ou massification? Paris, A. COLIN (Formation des enseignants), 1998.

BISENIUS-PENIN C. : « De la lecture cursive au café littéraire », in Le Français aujourd'hui, N $^{\circ} 133$ (Des réformes en pratiques),2001.

BOSMAN C, GÉRARD F.-M., ROEGIERS X. (éds.) : Quel avenir pour les compétencs ? Bruxelles, De Bœck \& Larcier (Pédagogies en développement), 2000.

BOURDIEU P. : Choses dites.Paris, Minuit (Le sens commun), 1987.

BOURDIEU P. : Méditations pascaliennes.Paris, Seuil (Liber), 1997.

BUCHETON D. : « Les postures du lecteur », in DEMOUGIN P. et MASSOL J.-F. : Lecture privée et lecture scolaire. La question de la littérature à l'école(Actes de la journée d'étude organisée à l'IUFM de Nîmes), 11 juin 1998. Grenoble, CRDP, 1999.

BURGOS M. : « Lecteurs experts, lecteurs convers. De quelques lectures en lycée professionnel et ailleurs ", inPratiques, $\mathrm{N}^{\circ} 76$ (L'interprétation des textes),1992.

BURGOS M. : « Lectures privées et lectures partagées », in Pratiques, $\mathrm{N}^{\circ} 80$ (Pratiques de lecteurs), 1993.

BURGOS M. : « La lecture des adolescents. Identification et interprétation », in L'École des Lettres, $\mathrm{N}^{\circ}$ 12-13 (Lire avec les adolescents),1994.

CANVAT K. : «Comprendre, expliquer, interpréter, décrire les textes littéraires. Postures de lecture et opérations métacognitives ", in Enjeux, $\mathrm{N}^{\circ} 46$ (Comprendre, interpréter, décrire... les textes littéraires),1999.

CANVAT K. : «Quels savoirs pour l'enseignement de la littérature ? Réflexions et propositions », in FOURTANIER M.-J., LANGLADE G. : Enseigner la littérature(Actes du colloque : Enjeux didactiques des théories du texte dans l'enseignement du français), 19-21 février 1998. Paris, Delagrave et CRDP Midi-Pyrénées (Savoir et Faire en Français), 2000a.

CANVAT K. : L'enseignement - apprentissage de la littérature au lycée. Objets, savoirs et compétences.Note de synthèse présentée pour l'habilitation à diriger des recherches. Université Charles de Gaulle - Lille 3, 2000 b.

CANVAT K., LEGROS G. : « Enseigner la poésie moderne ? », in Pratiques, $\mathrm{N}^{\circ} 93$ (Enseigner la poésie moderne),1997.

CARRÉ P. : « Pourquoi nous formons-nous ? », in Sciences humaines, Nº 92, 1999.

CHARLOT B. : Du rapport au savoir. Eléments pour une théorie. Paris, Anthropos, 1997.

CHEVALLARD Y. : La transposition didactique. Du savoir savant au savoir enseigné. Grenoble, La Pensée Sauvage, 1985.

CRAHAY M. : Peut-on lutter contre l'échec scolaire ?Bruxelles, De Bœck - Duculot (Pédagogies en développement), 1996.

DAUNAY B. : « La 'lecture littéraire' : les risques d'une mystification », in Recherches, $\mathrm{N}^{\circ} 30$ (parler des textes),1999a. 
DEVELAY M. : Donner du sens à l'école.Paris, ESF éditeur (Pratiques \& enjeux pédagogiques), 1996. DOLZ J., SCHNEUWLY B. : Pour un enseignement de l'oral. Initiation aux genres formels à l'école. Paris, ESF (Pédagogie), 1999.

DOLZ J., OLLAGNIER E. (EDS.) : Raisons éducatives, N 1-2(L'énigme de la compétence en éducation), 1999.

DORTIER J.-F : « Peut-on motiver autrui ? », in Sciences humaines, N 92, 1999.

DUFAYS J.-L. : « Les compétences littéraires en français langue maternelle ou première : état des lieux et essai de modélisation ", in COLLES L., DUFAYS J.-L., FABRY G., MAEDER C. : Didactique des langues romanes. Le développement de compétences chez l'apprenant (Actes du colloque de Louvain-la-Neuve), 27-29 JANVIER 2000. Bruxelles, De Bœck \& Larcier (Savoirs en pratique), 2001.

DUFAYS J.-L., GEMENNE L., LEDUR D. : Pour une lecture littéraire 1.Approches historique et théorique. Propositions pour la classe de français.Bruxelles, De Bœck \& Wesmael (Formation continuée), 1996.

DUMORTIER J.-L. : « Compétences de communication et savoirs littéraires. Regards du didacticien sur un référentiel », in La Lettre de la DFLM,N 25-2, 1999.

DUMORTIER J.-L. : « Formation littéraire et compétences de communication », in Enjeux, $\mathbf{N}^{\circ} 49$ (Enseignement de la littérature et compétences de communication), 2000.

DUMORTIER J.-L. : Lire le récit de fiction. Pour étayer un apprentissage : théorie et pratique. Bruxelles, De Bœck \& Wesmael (Savoirs en pratique), 2001.

GENETTE G. : L'œuvre de l'art, t. 2. La relation esthétique.Paris, Seuil (Poétique), 1997.

GENETTE G. : Figures IV. Paris, Seuil (Poétique), 1999.

JARRETY M. (dir.) : Propositions pour les enseignements littéraires.Paris, P.U.F., 2000.

JONNAERT P., VANDER BORGHT C. : Créer des conditions d'apprentissage.Bruxelles, De Bœck \& Larcier (Perspectives en éducation), 1999.

LAHIRE B. : Culture écrite et inégalités scolaires.Lyon, Presses universitaires de Lyon, 1993.

LAHIRE B. : La lecture populaire : les pratiques populaires de la lecture.Lyon, Voies livres (Pratiques et apprentissages de l'écrit, 54), 1994.

LE BOTERF G. : « De quel concept de compétence les entreprises et les administrations ont-elles besoin ? ", in BOSMAN C., GÉRARD F.-M., ROEGIERS X. (éds.) : Quel avenir pour les compétences ? BruxelleS, De Bœck \& Larcier (Pédagogies en développement), 2000.

MARTINAND J.-L. : Connaître et transformer la matière.Paris - Berne, Peter Lang, 1986.

MEIRIEU P. : Apprendre... oui, mais comment ?Paris, ESF éditeur (Pédagogies), 1988.

MEIRIEU P. : Le choix d'éduquer. Éthique et pédagogie.Paris, ESF éditeur (Pédagogies), 1991.

MEIRIEU P. : Frankenstein pédagogue. Paris, ESF éditeur (Pratiques \& enjeux pédagogiques), 1996.

PERRENOUD : Construire des compétences à l'École.Paris, ESF éditeur (Pédagogies), 1997.

PERRENOUD : «L'école saisie par les compétences », in BOSMAN C, GÉRARD F.-M., ROEGIERS X. (éds.) : Quel avenir pour les compétences ?Bruxelles, De Bœck \& Larcier (Pédagogies en développement), 2000.

PETITJEAN A. : «Écriture d'invention et enseignement de la littérature au lycée », in Enjeux, $\mathrm{N}^{\circ}$ 51-52 (Recherches en didactique de la littérature),2001. 
PETITJEAN A. : « L'écriture d'invention au lycée », in Pratiques, $\mathrm{N}^{\circ}$ 113-114 (La didactique de l'écriture), 2002.

PICARD M. : La lecture comme jeu. Paris, Minuit (Critique), 1986.

PRATIQUES, Nº 42 (L'écriture - imitation), 1984.

PRIVAT J.-M. : «L'institution des lecteurs », in Pratiques,N 80 (Pratiques de lecteurs),1993.

PRIVAT J.-M. : « Le rôle des sociabilités dans le développement du lecteur », in GARCIA-DEBANC C, GRANDATY M. et LIVA A. (dir.) : Didactique de la lecture. Regards croisés.ToulousE, Presses universitaires du Mirail (Questions à l'éducation), 1995.

PRIVAT J.-M. : « Culture littéraire et métalangages culturels », in BOUCHARD R., MEYER J.-C. (éds.) : Les métalangages de la classe de français(Actes du vi ${ }^{\mathrm{e}}$ colloque de la DFLM), 20-23 septembre 1995. Paris, DFLM, 1996.

PRIVAT J.-M., REUTER Y. (éds.) : Lectures et médiations culturelles (Actes du colloque de Villeurbanne). Lyon, Presses Universitaires de Lyon, 1991.

PRIVAT J.-M., VINSON M.-C. : « Habitat vertical et habituslectural », in Pratiques, $\mathrm{N}^{\circ} 52$ (Pratiques de lecture),1986.

PRIVAT J.-M., VINSON M.-C. : « Médiations culturelles et médiations textuelles au lycée », in Pratiques, $\mathrm{N}^{\circ}$ 107-108 (Les nouveaux programmes du lycée),2000.

PROST A. : L'enseignement s'est-il démocratisé ?Paris, P.U.F. (Sociologies), 1986.

REBIERE M. : « Une notion venue d'ailleurs... la posture », in BERNIE J.-P. (dir.) : Apprentissage, développement et significations.Bordeaux, Presses Universitaires de Bordeaux (Études sur l'éducation), 2001.

REUTER Y. : « La lecture littéraire : éléments de définition », in DUFAYS J.-L., GEMENNE L., LEDUR $D$ :Pour une lecture littéraire 2. Bilan et confrontations(Actes du colloque de Louvain-la-Neuve), 3-5 mai 1995. Bruxelles, De Bœck \& Wesmael (Formation continuée), 1996.

REY B. : Les compétences transversales en question.Paris, ESF éditeur (Pédagogies), 1996.

ROCHLITZ R. : Subersion et subvention. Art contemporain et argumentation esthétique.Paris, Gallimard (NRF Essais), 1994.

ROMAINVILLE M. : «L'irrésistible ascension du terme compétence en éducation 》, in Enjeux, $\mathrm{N}^{\circ}$ 37-38, 1996.

ROMAINVILLE M. : « Sous les réformes, les défis... ", in Wallonie. Revue du Conseil Économique et Social de la Région Wallonne, $\mathrm{N}^{\circ} 53,1998$.

ROMAINVILLE M. : « Les implications didactiques de l'approche par compétences », IN Enjeux, $\mathrm{N}^{\circ}$ 51-52, 2001.

ROUXEL A. : Enseigner la lecture littéraire.Rennes, Presses Universitaires de Rennes (Didact. français), 1997.

SCHAEFFER J.-M. : Pourquoi la fiction ?Paris, Seuil (Poétique), 1999.

TARDIF J. : Le transfert des apprentissages.Montréal (Canada), Les Éditions Logiques (Théories et pratiques dans l'enseignement), 1999.

TAUVERON C. (dir.) : Lire la littérature à l'école. Pourquoi et comment conduire cet apprentissage spécifique ? De la GS au CM. Paris, Hatier (Pédagogie), 2002. 
TERRISSE A. (éd.) : Didactique des disciplines. Les références au savoir.Bruxelles, De Bœck \& Larcier (Perspectives en éducation et formation), 2001.

\section{NOTES}

1. Les travaux de B. Rey, par exemple, montrent que la formation a des capacités générales est un leurre : la prégnance des contenus reste fondamentale (1996).

2. C'est la question célèbre de Fourier : «comment faire aimer les mathématiques à une jeune fille qui aime l'ail ?»

3. Ph. Meirieu a très bien décrit les dangers d'un projet éducatif qui se définirait comme projet de fabrication et de maîtrise de l'autre, de contrôle de son destin (1996). D'où la nécessité, pour l'enseignant, d'une «éthique de la retenue », qui permet, tout en utilisant la ruse, de laisser sa place à l'autre.

4. Cette expression a été introduite par J-L. Martinand, didacticien de la physique et de la technologie. Ce dernier invite à « mettre en relation les buts et contenus pédagogiques, en particulier les activités didactiques, avec les situations, les tâches et les qualifications d'une pratique donnée »(1986, p. 137).

5. En matière de pédagogie, observe perfidement (mais sans doute lucidement) J.-M. Albertini, c'est « l'économie qui mène le bal »...

6. Voir notamment l'ouvrage coordonné par M. Jarrety (2000).

7. Sur la question des pratiques sociales de référence, voir A. Terrisse, 2001.

8. La proposition surprendra peut-être ceux qui son habitués à opposer littérature et communication; elle confirmera peut-être aussi dans leur conviction les Cassandres qui déplorent "l'assassinat de la littérature ». Mais, si la littérature, "c'est ce qui s'enseigne », que fait-on lorsqu'on enseigne, sinon communiquer?

9. J.-M. Privat et M.-Ch. Vinson reformulent ainsi les droits imprescriptibles du lycéen lecteur de l'an 2000 :

Aller lui-même acheter ses livres en librairie (ou les commander par Internet).

Débattre de ses lectures avec des pairs ou des (cyber-)experts.

S'entretenir de ses pratiques culturelles avec ses professeurs et le documentaliste.

Être régulièrement confronté à l'actualité médiatique sur les livres.

Lire de la littérature fraîche au moins une fois par trimestre.

Lire ou relire des livres « faciles » ou qui ne son plus de son age...

Noter des commentaires personnels directement sur la page au fil de la lecture.

Construire des arts de faire pour s'approprier des textes longs ou difficiles

Savoir arrêter et reprendre une lecture sans problème.

Apprendre à intervenir comme co-auteur dans des textes électroniques. (2000, p. 219).

10. Voir notamment G. Genette, 1997.

\section{AUTEUR}

KARL CANVAT

IUFM Lorraine (Nancy) 